EPJ Web of Conferences 73, 06002 (2014)

DOI: $10.1051 /$ epjconf/20147306002

(C) Owned by the authors, published by EDP Sciences, 2014

\title{
Few-nucleon system dynamics in medium energy domain
}

\author{
H. Machner ${ }^{\mathrm{a}}$
}

Fakultät für Physik, Universität Duisburg-Essen, Lotharstr. 1, 47048 Duisburg, Germany

\begin{abstract}
Nucleon-nucleon interactions and hyperon nucleon interactions are study in three body final state with one being a meson. In addition to final state parameters possible resonances in the two baryon states are of interest.
\end{abstract}

\section{Introduction}

An excellent tool to study baryon-baryon interaction is the investigation of the $B_{a} B_{b} \rightarrow m B_{1} B_{2}$ reaction with $m$ being a meson. This meson assisted $f s i$ is favourable with respect to three baryon states, which could show up contributions of three body forces. Since the meson-baryon interaction is much weaker than the baryon-baryon interaction the factorisation of the $S$-wave scattering amplitude $f_{s}$ into a mean matrix element $T$ and a final state interaction $f s i$

$$
\frac{d^{2} \sigma}{d \Omega_{m} d M_{B_{1} B_{2}}}=\left|f_{s}\right|^{2} \Phi_{3}=|T|^{2} \frac{q^{2}+\beta_{s}^{2}}{q^{2}+\alpha_{s}^{2}} \Phi_{3}
$$

is a good approximation. With $\phi_{3}$ the three body phase space is denoted. The fraction is the $f s i$ in the inverse Jost function approximation. In cases where singlet and triplet spin states are possible we will apply two different scattering amplitudes. Here we will discuss only nucleon-nucleon interaction in $p p \rightarrow \pi N N$ pion production and hyperon-nucleon interaction in $p p \rightarrow K^{+} Y N$ associated kaon production.

\section{Nucleon-nucleon interaction}

The $p p$ interaction seemed be well under control. However in a study of $p+p \rightarrow \pi^{0}+p+p$ reactions close to threshold, where only $S s$ wave contributes, the shape of the excitation function should be determined by the phase space and $f s i$. The Bloomington group [1] found the necessity of using a scattering length $a_{p p}=-1.5 \mathrm{fm}$ instead of the accepted value of $a_{p p}=-7.82 \pm 0.01 \mathrm{fm}$. They claim that "this indicates clearly that factorising the $f s i$ of the protons and neglecting all other distortions in the initial and final states is only an approximation". Since the analysis made is not without theory input, the GEM collaboration at COSY [2] decided to repeat the experiment well above threshold and extract partial waves. The result of the study was that the scattering length from $p p$ elastic scattering is the

\footnotetext{
a e-mail: hartmut.machner@uni-due.de
}

This is an Open Access article distributed under the terms of the Creative Commons Attribution License 4.0, which permits unrestricted use, distribution, and reproduction in any medium, provided the original work is properly cited. 

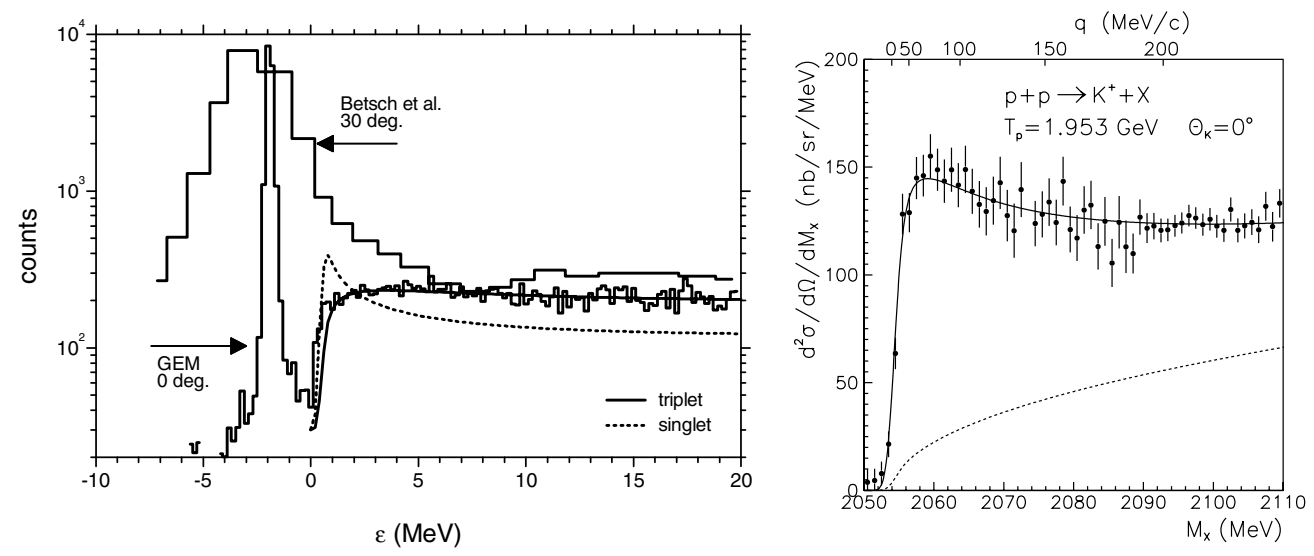

Figure 1. Left: missing mass spectra for $p n$. The upper data are from [5], the lower from [3]. Also shown are fits for the triplet state and the singlet state. Right: missing mass spectrum for $\Lambda p$ system taken at forward direction at the indicated beam energy. The solid curve is the fit with Eq. (1) to the data, the dotted curve is pure phase space without $f$ si.

same as in $f s i$ when the $S s$ wave part is properly extracted from the data. The $\Delta$ has been included in the $P p$ wave and was found to be a dominating process.

It is a long standing problem how the singlet and triplet strength of $p n f s i$ can be disentangled. Similar to the deuteron, the triplet state, there should be a bound singlet state. However, such a state has never been observed. But there should be an unbound pole at $68 \mathrm{keV}$. It should be mentioned that a total angular momentum $J=1$ triplet state can be constructed in two different ways: $J_{z}=L_{z}+S_{z}=0+1$ or $J_{z}=L_{z}+S_{z}=2-1$. The latter is the well known $D$-state of the deuteron which an amplitude of $\approx 20 \%$ of the total amplitude.

The GEM collaboration [3] performed a high resolution study of the reactions $p+p \rightarrow \pi^{+}+X^{+}$ with $X^{+}=d$ and $X^{+}=p+n$ at a beam momentum of $1642.5 \mathrm{MeV} / \mathrm{c}$. The pion was detected in the forward direction by the magnetic spectrograph Big Karl [4]. The resulting spectrum is shown in Fig. 1. A narrow peak at $\epsilon=-2.22 \mathrm{MeV}$, the deuteron, is well separated from the $p n$ continuum. The remaining background is mainly due to muons, which could not be separated from pions. The spectrum is compared to one from [5] taken at $954 \mathrm{MeV} / \mathrm{c}$ at an angular range $25-40$ degrees with scintillating detectors. In this case the separation of deuterons and $p n$ continuum is less clear. Also shown are fits with $f s i$ parameters from elastic scattering and only the matrix element is fitted. The contribution of spin singlet is negligible.

\section{Hyperon-nucleon interactions}

In this part we will study only the $\Lambda p$ system, which is very much like the $n p$ system. The $\Lambda p$ scattering has been observed in bubble chambers. The bubble chamber method suffers from the meager statistics. In addition, statistical error bars are largest for the smallest $\Lambda$ momenta. However, this range is the only one which is sensitive to effective range parameters. Again, smaller energies are provided by a three body final state $p+p \rightarrow K^{+}+\Lambda+p$ known as associated strangeness production. The HIRES collaboration measured this reaction in a high resolution experiment again making use of the magnetic spectrograph Big Karl [6] at $T_{p}=1.953 \mathrm{GeV}$ and $2.0812 \mathrm{GeV}$. Two threshold Cerenkov counters were applied in the focal plane in order to suppress pion counts [7]. The obtained spectrum is shown in Fig. 1. Similar to the $p n$ system spin singlet and triplet were fitted. So altogether six free parameters have to be adjusted. Finally three body final state and elastic scattering cross sections were fitted simultaneously 

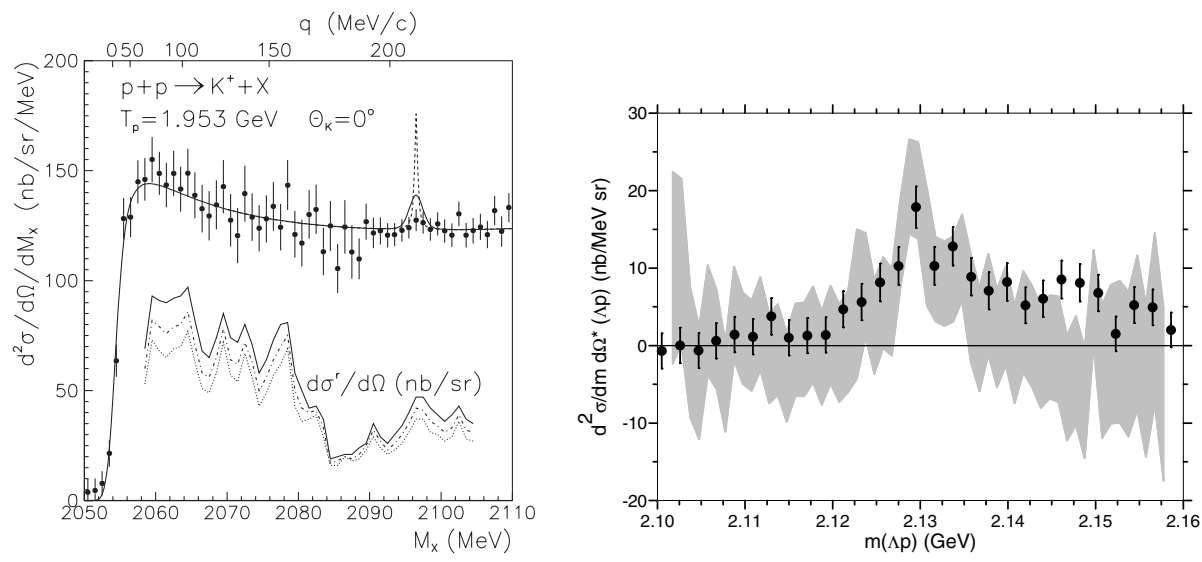

Figure 2. Left panel: missing mass spectrum of the reaction $p+p \rightarrow K^{+}+X$ with $X=(\Lambda p)$. The upper axis indicates the c.m. momentum $q$ of the $\Lambda p$ system. Solid line: FSI fit curve with resonance excursion excluded by the $\chi^{2}$ test. Dashed line: Same resonance excursion without folding with the effective resolution function. Resonance parameters: $\mathrm{d} \sigma^{r} / \mathrm{d} \Omega_{K}=42 \mathrm{nb} / \mathrm{sr}, M_{r}=2096.5 \mathrm{MeV}, \Gamma=500 \mathrm{keV}$. The lower curves represent upper limits of the production cross section $\mathrm{d} \sigma^{r} / \mathrm{d} \Omega_{K}(\mathrm{nb} / \mathrm{sr}$ ) are indicated for $\Gamma=100 \mathrm{keV}$ (dotted line), $\Gamma=500 \mathrm{kev}$ (dasheddotted line) and $\Gamma=1.0 \mathrm{MeV}$ (solid line). Right panel: spectra of the $\Lambda p$ system close to the $\Sigma N$ threshold. The shaded data are from [8] including uncertainties, dots with error bars are from [9]. From the inclusive data from [10] contributions from $\Sigma^{+} n$ and $\Sigma^{0} p$ were subtracted. From both data sets phase space distributions including $f s i$ for the non-resonant cross section were subtracted.

Figure 3. $\Sigma N$ threshold regions from different data (see Ref. [8]) and Breit-Wigner fits. Nonresonant cross section has been subtracted. The errors indicate the thresholds. The data are from Refs. [14-17], [11], and [9].

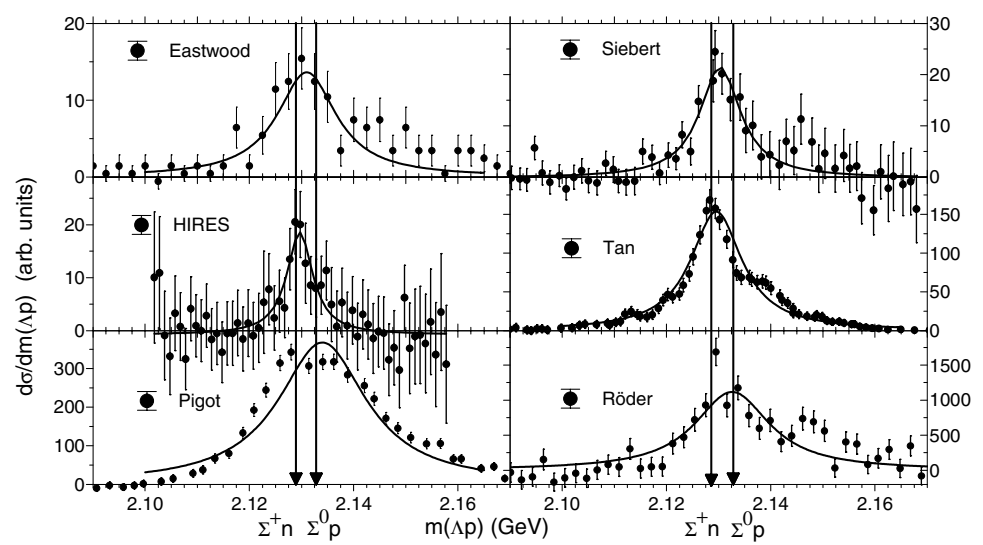

adding the effective range parameters obtained by [11] for triplet scattering. The final results for the effective range parameters are $a_{s}=-2.42_{-0.25}^{+0.16}, r_{s}=2.21_{-0.36}^{+0.16}, a_{t}=-1.56_{-0.22}^{+0.19}$, and $r_{t}=3.7_{-0.6}^{+0.6}$ all measured in $\mathrm{fm}$.

There might be a $S=1$ dibaryon which would show up as a narrow resonance in the $\Lambda p$ channel. Aerts and Dover [12] predicted a spin singlet dibaryon $D_{s}$ between the $\Lambda p$ and the $\Sigma N$ thresholds. We searched for such a resonance in $\Lambda p$ elastic scattering as well as in FSI (Ref. [13]). We assumed a resonance at $42.5 \mathrm{MeV}$ having a width of $0.50 \mathrm{MeV}$. Such a resonance could, however, not been seen in the elastic cross sections. This resonance corresponds to a mass of $2.0965 \pm 0.5000 \mathrm{MeV} / \mathrm{c}^{2}$ in FSI. In a previous experiment [14] some structure had been observed. This is not confirmed by the high resolution experiment [13]. A $\chi^{2}$ analysis leads to upper limits for the cross sections for the $p p \rightarrow K^{+} D_{s}$ reaction (see right panel in Fig. 2). 
However, a broad peak occurs in the $\Lambda p$ system close to the $\Sigma N$ thresholds (see right panel in Fig. 2 and Fig. 3). It is also visible in the reaction $K^{-} d \rightarrow \pi^{-} \Lambda p$. It can be either a cusp or a bound deuteron like $\Sigma N$ or a resonance in the $\Lambda p$ system. The imposition of analyticity requires that the presence of the $\Sigma^{+} n$ channel must be felt below threshold as well, thus creating the rapid decrease in the $\Lambda p$ cross section on the low-mass side of the peak. Fits within the Flatté approach are bad thus making the assumption of a cusp unlikely. If the structure would be to a $\Sigma N$ deuteron, its centroid has to be below the corresponding threshold by the amount of the binding energy. However, the position extracted by fitting Breit-Wigner distributions to the data vary to much to come to conclusive results.

The author is grateful to his colleagues from the GEM and HIRES collaborations at COSY. He thanks the Organisers of the Conference MENU 2013 for their kind hospitality and for supporting financially his participation in this conference.

\section{References}

[1] H.O. Meyer, et al., Phys. Rev. C 63, 064002 (2001)

[2] M. Betigeri, et al., Phys. Rev. C 65, 064001 (2002)

[3] M. Abdel-Bary, et al. (GEM Collaboration), Phys. Lett. B 619, 281 (2005)

[4] H. Bojowald, et al., Nucl. Instrum. Methods Phys. Res. A 487, 314 (2002)

[5] A. Betsch, et al, Phys. Lett. B 446, 179 (1999)

[6] M. Drochner, et al., Nucl. Phys. A 643, 55 (1998)

[7] R. Siudak, et al., Nucl. Inst. \& Meth. in Phys. Res. A 596, 311 (2008)

[8] H. Machner, J. Haidenbauer, F. Hinterberger, A. Magiera, J. Niskanen, J. Ritman, R. Siudak, Nucl. Phys. A 901, 65 (2013)

[9] M. Röder, et al., Eur. Phys. J. A 49, 157 (2013)

[10] A. Budzanowski, et al. (HIRES Collaboration), Phys. Lett. B 687, 31 (2010)

[11] T.H. Tan, Phys. Rev. Lett. 23, 395 (1969)

[12] A.T.M. Aerts, C.B. Dover, Nucl. Phys. B 253, 116 (1985)

[13] A. Budzanowski, et al. (HIRES Collaboration), Phys. Rev. D 84, 032002 (2011)

[14] R. Siebert, et al., Nucl. Phys. A 567, 819 (1994)

[15] D. Eastwood, J.R. Fry, F.R. Heathcote, G.S. Islam, D.J. Candlin, G. Copley, G.R. Evans, J.R. Campbell, W.T. Morton, P.J. Negus et al., Phys. Rev. D 3, 2603 (1971)

[16] A. Budzanowski, et al. (HIRES Collaboration), Phys. Lett. B 692, 10 (2010)

[17] C. Pigot, et al., Nucl. Phys. B 249, 172 (1985) 